\title{
Review: risk for suicide is increased for most mental disorders where patients require treatment in a hospital setting
}

\author{
Harris EC, Barraclough B. Suicide as an outcome for mental disorders. A meta-analysis. Br J Psychiatry 1997 Mar;170:205-28.
}

\section{Objective}

To determine the risk for suicide among patients with common mental disorders.

\section{Data sources}

English language studies were identified by searching Index Medicus and Medline (1966 to 1993) using the terms mental disorders, mortality, follow up, brain injury, eating disorders, epilepsy, suicide attempt, and psychosurgery. Additional studies were identified by reviewing several journals up to mid 1995 and by scanning the reference lists of retrieved articles.

\section{Study selection}

Studies were selected if they reported the mortality of mental disorders, had $\geqslant 2$ years of follow up, had $<10 \%$ loss to follow up, and compared observed numbers of suicides with those expected.

\section{Data extraction}

Data were extracted on the mental disorder studied, sample size, length of follow up, country where the investigation occurred, observed suicide rates, and expected suicide rates, if provided in the article. If the expected suicide rates were not provided, they were estimated using the World Health Organisation statistical reports for the relevant country and years, combined with the age and sex composition and mean observation period for each study.

\section{Main results}

249 studies met the selection criteria. Standardised mortality ratios (SMRs)* were calculated for each disorder by comparing the sums of their observed and expected values. Of 44 disorders considered, 36 had a statistically significant raised SMR for suicide, 5 had a raised SMR that did not reach statistical significance, 1 SMR was not raised, and 2 SMRs could not be calculated because no suicides were reported. It appeared that there was an increased risk for suicide for most mental disorders where patients required treatment, mainly in a hospital setting, except for those with mental retardation, dementia, and agoraphobia. When disorders were grouped by their cause, organic disorders had the lowest SMR (332, 95\% CI 293 to 375, ranging from an SMR of 88 for dementia not otherwise specified to an SMR of 8750 for surgically treated temporal lobe epilepsy); substance abuse was next (SMR 574, CI 541 to 609, ranging from an SMR of 244 for smoking [tobacco] to an SMR of 4423 for sedatives, with other drugs); and functional disorders had the highest SMR (1209, CI 1178 to 1241, ranging from an SMR of 372 for neurosis to an SMR of 5714 for stupor). Risk for suicide appeared to be highest at the beginning of treatment and diminished thereafter.

\section{Conclusion}

The risk for suicide is increased for most mental disorders where patients require treatment, mainly in a hospital setting, except for those with mental retardation, dementia, and agoraphobia.

*A standardised mortality ratio is a way of comparing 2 groups while adjusting for differences between the groups (eg, adjusting for age and sex differences in a sample compared with the population).

Source of funding: in part, Knoll Pharmaceuticals.

For article reprint: not available

Article and commentary also published in Evidence-Based Medicine 1997 Sep-Oct;2:156.

\section{Commentary}

This study is the corollary of the 1974 investigation by Barraclough et al of 100 consecutive patients who committed suicide $^{1}$; the investigators used the psychological autopsy method (a retrospective reconstruction of the life, personality, and state of mind of the deceased person from records and interviews with others). $93 \%$ of the individuals who committed suicide had been clinically ill, and 64\% had uncomplicated depressive illness. This study by Harris and Barraclough approaches the problem from the opposite direction, finding increased risk for suicide in 36 of 44 disorders, with functional disorders collectively having the highest risks. However, within the functional disorders group, wide variability exists, ranging from a high of 20 times greater than that expected for major depression to a low of 4 in neurosis. Other functional disorders, such as bipolar disorder, brief reactive psychosis, schizophrenia, adjustment disorders, dysthymia, and anxiety disorders, also carry substantial risk for suicide.

The importance of this study for the clinician is that the possibility of a suicidal outcome must be considered in almost every condition. The clinician should routinely inquire during the initial assessment about the presence of depression (as a symptom), future perspective, and about negative (including suicidal) thinking. Further assessments are needed from time to time, especially after hospital discharge, when there is a turn for the worse, or the chronicity and resistance to treatment might induce hopelessness in the patient (and physician). This counsel also applies to medical disorders with links to mental disorders, such as substance abuse, HIV and AIDS, cancer, Huntington's disease, multiple sclerosis, peptic ulcer, renal dialysis, spinal cord injury, and systemic lupus erythematosus. ${ }^{2}$

This important study quantifies the risk for suicide for common mental disorders. Pooling data does carry the risk for reductionism, minimising heterogeneity of samples, and variability between studies. Older material may also affect findings in unknown ways.

Isaac Sakinofsky, MD Clarke Institute of Psychiatry Toronto, Ontario, Canada

1 Barraclough BM, Bunch J, Nelson B, et al. A hundred cases of suicide: clinical aspects. $\mathrm{Br} J$ Psychiatry 1974;125:355-73.

2 Harris EC, Barraclough BM. Suicide as an outcome for medical disorders. Medicine 1994;73:
281-96. 